\title{
Expression of a Kinesin-Related Motor Protein Induces Sf9 Cells to Form Dendrite-Like Processes with Nonuniform Microtubule Polarity Orientation
}

\author{
David J. Sharp, ${ }^{1}$ Ryoko Kuriyama, ${ }^{2}$ and Peter W. Baas ${ }^{1}$ \\ ${ }^{1}$ Department of Anatomy and Program in Neuroscience, The University of Wisconsin Medical School, Madison, \\ Wisconsin 53706, and 'Department of Cell Biology and Neuroanatomy, The University of Minnesota Medical School, \\ Minneapolis, Minnesota 55455
}

The microtubules (MTs) within neuronal processes are highly organized with regard to their polarity and yet are not attached to any detectable nucleating structure. Axonal MTs are uniformly oriented with their plus ends distal to the cell body, whereas dendritic MTs are of both orientations. Here, we sought to test the capacity of motor-driven MT transport to organize distinct MT patterns during process outgrowth. We focused on CHO1/MKLP1, a kinesin-related protein present in the midzonal region of the mitotic spindle where MTs of opposite orientation overlap. Insect ovarian Sf9 cells induced to express the $\mathrm{N}$-terminal portion of the molecule form MT-rich processes with a morphology similar to that of neuronal dendrites (Kuriyama et al., 1994). Nascent processes contain uniformly plus-end-distal MTs, but these are joined by minus-enddistal MTs as the processes continue to develop. Thus, this
CHO1/MKLP1 fragment establishes a nonuniform MT polarity pattern and does so by a similar sequence of events as occurs with the dendrite, the antecedent of which is a short process with a uniform MT polarity orientation. Two lines of evidence suggest that these results are elicited by motor-driven MT transport. First, there is a depletion of MTs from the cell body during process outgrowth. Second, the same polarity pattern is obtained when net MT assembly is suppressed pharmacologically during process formation. Collectively, these findings provide precedent for the idea that motor-driven transport can organize MTs into distinct patterns of polarity orientation during process outgrowth.

Key words: microtubule; kinesin; dendrite; neuron; mitosis; spindle
The polarity orientation of microtubules (MTs) within a cell is fundamental to the organization of its cytoplasm and for regulating features of its architecture and motility. In most cell types, MTs are organized relative to their polarity by direct attachment of the minus ends of the MTs to their nucleation sites at the centrosome or basal body (Heidemann and McIntosh, 1980; Euteneuer and McIntosh, 1981). However, neurons generate elongate processes containing dense arrays of MTs that are not attached to any detectable nucleating structures and, quite remarkably, these MT arrays are characterized by sophisticated patterns of polarity orientation. In the axon, MTs are uniformly oriented with their plus ends distal to the cell body (Burton and Paige, 1981; Heidemann et al., 1981; Baas et al., 1987, 1988). In dendrites, an initial population of plus-end-distal MTs is joined by a population of minus-end-distal MTs, resulting in a nonuniform polarity pattern (Baas et al., 1988, 1989). Recent studies from our laboratory indicate that these MT polarity patterns are established under experimental conditions that suppress MT assembly but not MT transport (Baas and Ahmad, 1993; Sharp et al., 1995). On the

Received March 1, 1996; revised April 23, 1996; accepted April 29, 1996.

This work was supported by grants from National Institutes of Health (NIH) and the National Science Foundation to P.W.B., and from NIH and the Council for Tobacco Research to R.K. P.W.B. is the recipient of a Research Career Development Award from NIH. D.J.S. is supported in part by a grant from NIH to the Neuroscience Program at The University of Wisconsin. We thank Wenqian Yu, Matthew Schwei, and Kathryn Bollinger for assistance. We especially thank Dr. Scott Brady for helpful discussions about motor proteins.

Correspondence should be addressed to Dr. Peter W. Baas, Department of Anatomy and Program in Neuroscience, The University of Wisconsin Medical School, 1300 University Avenue, Madison, WI 53706.

Copyright (C) 1996 Society for Neuroscience 0270-6474/96/164370-06\$05.00/0 basis of these results, we proposed that specific molecular motors interact with MTs destined for axons or dendrites, transporting them into the processes with the appropriate orientation.

Precedent for motor proteins mediating interactions between MTs derives from the mitotic spindle (Hoyt, 1994; McIntosh, 1994). One such motor is the kinesin-related protein CHO1/ MKLP1, a major component of the mammalian mitotic spindle (Sellitto and Kuriyama, 1988; Nislow et al., 1992). This motor localizes near the centrosome early in mitosis, but then concentrates later in mitosis in the midzonal region where antiparallel MTs from opposite poles overlap. Studies of the properties of CHO1/MKLP1 in vitro indicate that it transports MTs relative to one another. Specifically, it transports MTs with minus ends leading toward the plus ends of other MTs (Nislow et al., 1992). This finding, together with the fact that function-blocking antibodies to CHO1/MKLP1 arrest mitotic progression (Nislow et al., 1990), strongly suggests that CHO1/MKLP1 transports antiparallel MTs apart during spindle elongation.

Recent studies of living cells provide support for the capacity of CHO1/MKLP1 to organize MTs. These studies demonstrate that ectopic expression of approximately half of the molecule at the $\mathrm{N}$ terminus can induce normally rounded insect ovarian Sf9 cells to form MT-rich processes (Kuriyama et al., 1994). Interestingly, these processes have a thick tapering morphology similar to that of dendrites. These findings, together with the observation that CHO1/MKLP1 transports oppositely oriented MTs against one another in vitro, suggest the compelling possibility that the $\mathrm{N}$-terminal portion of CHO1/MKLP1 may organize MTs in these processes into arrays with a nonuniform polarity pattern similar to 
that of dendrites. Evidence to this effect would provide direct documentation of the capacity of a motor protein to organize MTs into arrays with distinct polarity patterns such as those found in the neuron.

\section{MATERIALS AND METHODS}

Cell culture and baculovirus infection. Sf9 cells were maintained in TNM-FH medium, which consists of Grace medium obtained from Gibco (Grand Island, NY), $0.33 \%$ yeastolate, $0.33 \%$ lactalbumin hydrolysate, and $10 \%$ fetal bovine serum, pH 6.2 (Kuriyama et al., 1994). The cells were maintained in plastic tissue culture flasks and then subcultured onto glass coverslips for baculovirus infection and subsequent experimental analyses. Before plating, the glass coverslips had been pretreated with polylysine (Sigma, St. Louis, MO) for immunofluorescence analyses or with vectabond (Vector Labs, Burlingame, CA) for MT polarity analyses. Baculovirus constructs encoding approximately half the molecule including the $\mathrm{N}$-terminal region were generated as described previously (Kuriyama et al., 1994). Medium containing the viral particles was added directly to plated cells, and this resulted in a nearly $100 \%$ infection rate as determined by immunofluorescence staining for the $\mathrm{N}$-terminal region of CHO1/MKLP1 and by appearance of viral particles in electron micrographs.

Immunofluorescence microscopy. Immunofluorescence staining of Sf9 cells was performed using one of two methods. The first method was as described previously (Kuriyama et al., 1994). Briefly, cells were fixed for $5 \mathrm{~min}$ in cold methanol $\left(-20^{\circ} \mathrm{C}\right)$, transferred to cold absolute acetone $\left(-20^{\circ} \mathrm{C}\right)$ for $5 \mathrm{~min}$, air dried, and then rehydrated in PBS containing $0.05 \%$ Tween-20. The cells then were exposed either to a mouse monoclonal $\beta$-tubulin antibody (Amersham, Arlington Heights, IL) or to a rabbit polyclonal antiserum that recognizes sites within the N-terminal half of CHO1/MKLP1 (Kuriyama et al., 1994). In the second method, cells were preextracted under conditions that remove free tubulin but stabilize existing MTs, and then fixed as described previously (Sharp et al., 1995). The cells then were exposed to the mouse monoclonal $\beta$-tubulin antibody noted above. At this point, cultures prepared by these methods were rinsed free of primary antibodies and exposed to appropriate fluorescent secondary antibodies as described previously (Kuriyama et al., 1994; Sharp et al., 1995). For quantitative analyses on MT levels in the preextracted cells, images were captured with the Zeiss LSM 410 Laser Confocal Microscope (Zeiss, Thornwood, NY). The pinhole was opened minimally to allow the highest resolution of fluorescently labeled proteins, serial optical sections were taken through the entire cell, and the images then were reconstructed by Zeiss system software. This resulted in complete visualization of fluorescently labeled material in a single high-resolution image. Fluorescence intensities were quantified using National Institutes of Health (NIH) Image software (provided free of charge from the NIH, Bethesda, MD). Fluorescence intensities were calculated for uninfected cells, infected process-bearing cells, and infected cells that grew processes in the presence of vinblastine sulfate (see below), and expressed in arbitrary fluorescence units (AFUs). Ten cells were analyzed for each of these conditions, and statistical analyses were performed using the Student's $t$ test.

MT polarity studies. MT polarity analyses were performed by a modification of methods described previously (Heidemann and McIntosh, 1981; Euteneuer and McIntosh, 1982; Baas et al., 1987, 1988, 1989, 1991; Maekawa et al., 1991; Chen et al., 1992; Baas and Ahmad, 1993; Sharp et al., 1995). This method involves the decoration of existing MTs with exogenous brain tubulin using a buffer that promotes the formation of lateral protofilament sheets. These sheets appear as curved appendages called "hooks" on the MTs when viewed in cross-section under the electron microscope. A clockwise hook indicates that the plus end of the MT is directed toward the observer, whereas a counterclockwise hook indicates that the minus end is directed toward the observer. Cultures were rinsed briefly in PBS and then incubated at $37^{\circ} \mathrm{C}$ for $20 \mathrm{~min}$ in a solution containing $0.25 \%$ saponin, 0.5 M Pipes, 0.1 M EGTA, $0.01 \mathrm{~mm}$ EDTA, $0.1 \mathrm{~mm} \mathrm{MgCl} 2,2.5 \%$ DMSO, $0.5 \mathrm{~mm}$ GTP, and $1.2 \mathrm{mg} / \mathrm{ml}$ brain tubulin. Cultures then were fixed by the addition of an equal quantity of $4 \%$ glutaraldehyde and then processed and embedded for electron microscopy by conventional methods. The extraction conditions resulted in some splaying apart of the MTs, permitting us to interpret accurately the polarity orientation of MTs within normally tight bundles. Video-print images were obtained before sectioning, and these were used to document precisely the points along the lengths of processes at which cross-
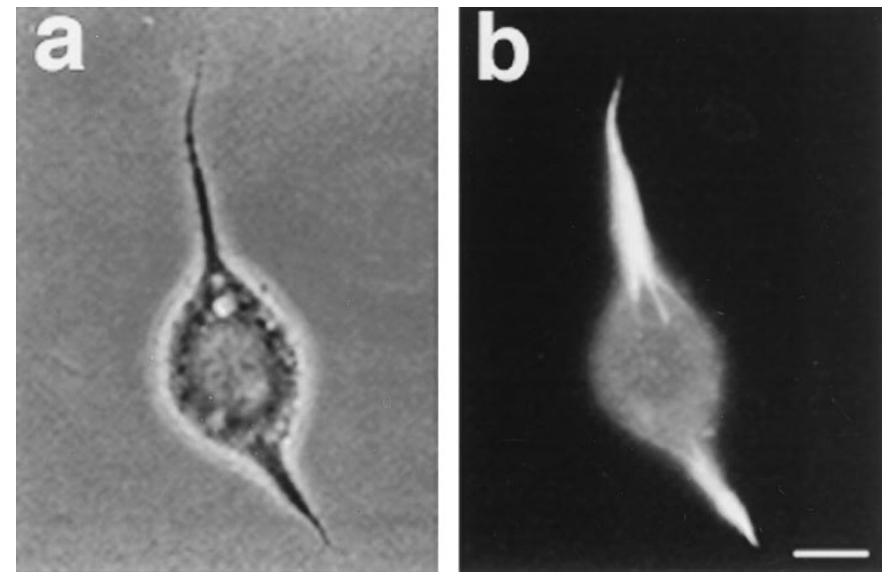

Figure 1. a, Phase-contrast micrograph of a moth ovarian Sf9 cell $3 \mathrm{~d}$ after infection with a baculovirus construct encoding the $\mathrm{N}$-terminal half (containing the motor domain) of CHO1/MKLP1. $b$, Same cell immunostained for tubulin. Within $3 \mathrm{~d}$ of infection, up to $40 \%$ of infected cells had extended at least one process, and a portion had extended several. In the process-bearing cells, tubulin staining was concentrated within the processes. Scale bar, $8 \mu \mathrm{m}$.

sections were made. The sections were visualized and photographed using a JEOL CX100 electron microscope (JEOL USA, Peabody, MA).

Pharmacological studies. In one set of experiments, MT assembly was suppressed by the addition of vinblastine sulfate (Sigma) to cultures at a final concentration of $10 \mathrm{~nm}$. Vinblastine was added at the time of baculovirus infection.

\section{RESULTS}

Insect ovarian Sf9 cells normally are spherical in shape, do not extend cytoplasmic processes, and stain diffusely for tubulin in immunofluorescence analyses (Knops et al., 1991; Kuriyama et al., 1994). Marked alterations occur within $3 \mathrm{~d}$ of exposure to baculovirus vectors coding for approximately half of the CHO1/ MKLP1 molecule containing the $\mathrm{N}$-terminal motor domain. These alterations include changes in the pattern of tubulin staining and the outgrowth of thick tapering processes from a portion of the cells (Kuriyama et al., 1994). In the infected cells that remain spherical, tubulin staining is manifested as a circumferential ring just under the plasma membrane. In the process-bearing cells, tubulin staining is concentrated within the processes, leaving the cell body nearly devoid of staining. Cells induced to express the entire motor form processes on occasion, but the proportion of process-bearing cells is significantly lower (Kuriyama et al., 1994). For this reason, we focused our present efforts on expression of the $\mathrm{N}$-terminal portion, which resulted in the formation of processes from 10 to $40 \%$ of the infected cells. Figure 1, $a$ and $b$, shows phase-contrast and immunofluorescence images, respectively, of a process-bearing cell. The concentration of tubulin staining within the process and its thick tapering morphology are apparent. Immunostains with polyclonal antiserum raised against the N-terminal half of the CHO1/MKLP1 molecule show high levels of the motor throughout the cell body and processes (data not shown), indicating the presence of the motor both in MT-rich and MT-depleted regions of the cell.

\section{MT polarity analyses}

Our principal goal was to determine the pattern of MT polarity orientation established within the motor-induced processes. To determine the orientation of the MTs within the processes, we used the standard hooking assay for MT polarity determination 

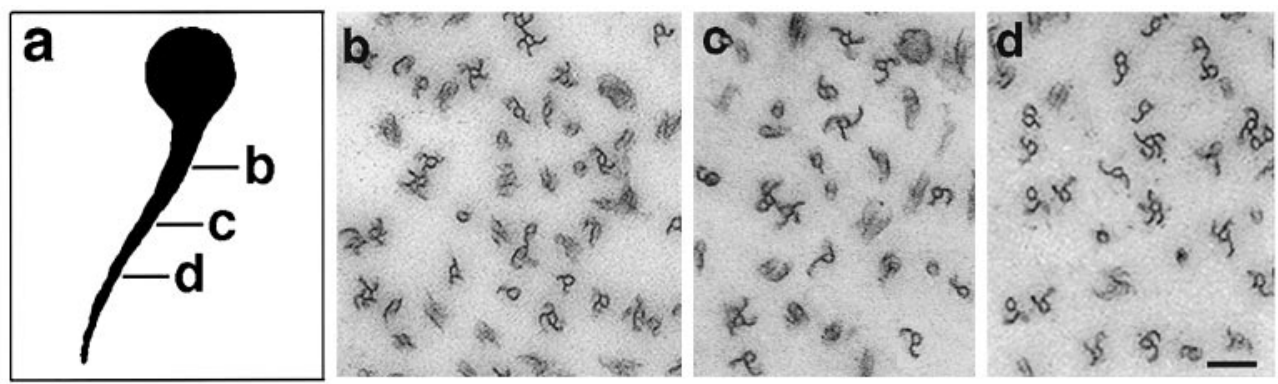

tended a thick tapering process after being induced to express the $\mathrm{N}$-terminal half of CHO1/MKLP1. Arrows indicate points along the length of the process at which MT polarity orientation was determined. $b-d$, Electron micrographs of process cross-sections at points indicated in $a$. Clockwise hooks indicate MTs oriented with their plus ends distal to the cell body, whereas counterclockwise hooks indicated MTs with their minus ends distal to the cell body. $e$, Schematic showing the MT polarity data obtained from six processes analyzed at multiple points along their lengths. All cells chosen for analysis had extended a single process $35-50 \mu \mathrm{m}$ in length. Four additional processes were analyzed at their midpoints only and produced results consistent with those shown here. $\mathrm{Nu}$ merals above arrows indicate the fraction of counterclockwise to clockwise hooks counted per region and the percentage of counterclockwise hooks. The proportion of hooked MTs was high, nearly $90 \%$, and of these, only $\sim 15 \%$ were ambiguous. Scale bars: $a, 10 \mu \mathrm{m} ; b-d, 0.15$ $\mu \mathrm{m} ; e, 10 \mu \mathrm{m}$.

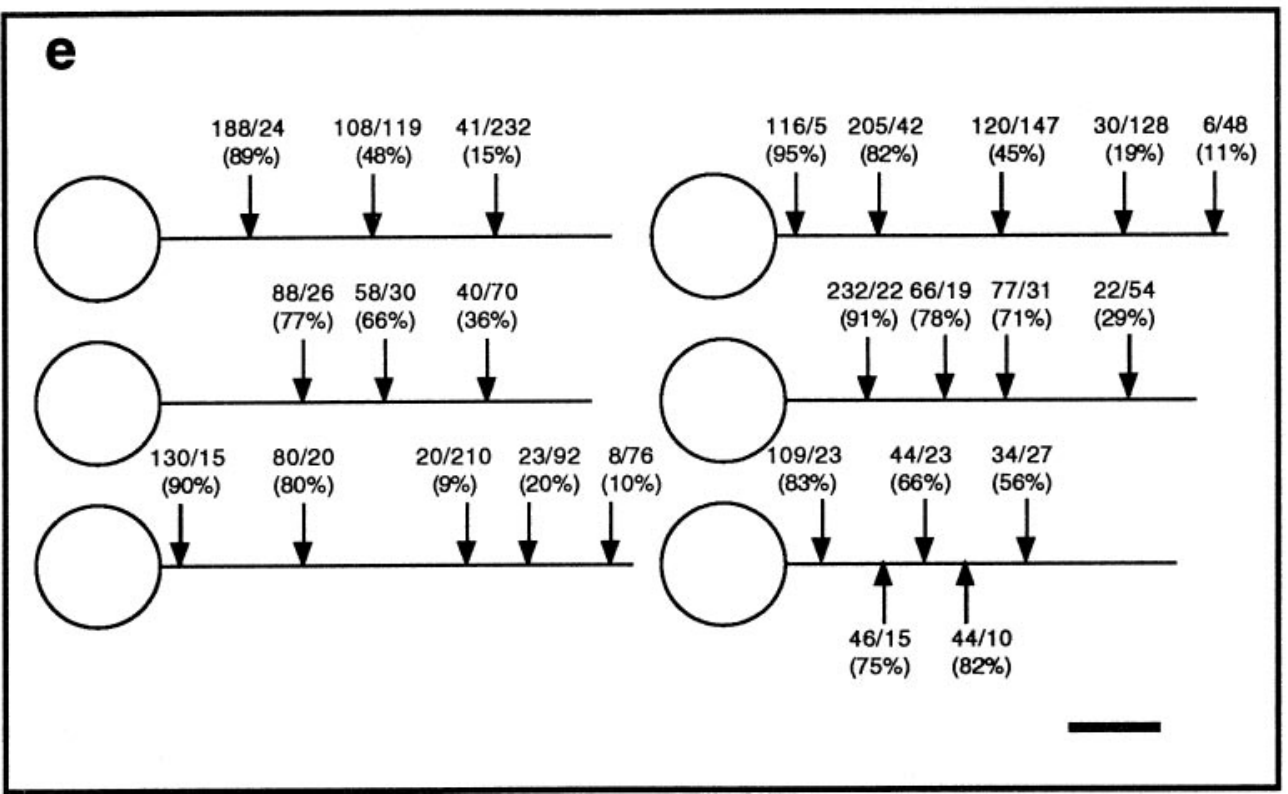

(see Materials and Methods). In this method, cells are extracted in the presence of exogenous brain tubulin in a buffer that promotes the formation of hooked appendages on the MTs. All hooks were viewed from the vantage point of the distal tip of the process such that clockwise hooks indicate MTs with their plus ends distal to the cell body and counterclockwise hooks indicate MTs with their minus ends distal to the cell body. Ten processes, 35-50 $\mu \mathrm{m}$ in length, were analyzed at their midpoints, and six of these were analyzed at additional points along their lengths. Figure 2 shows three points from one of these processes. The midregion contained approximately equal numbers of MTs of each orientation (Fig. 2c), distal regions contained predominantly plus-end-distal MTs (Fig. 2d), whereas regions near the cell body contained predominantly minus-end-distal MTs (Fig. 2b). All processes analyzed showed this same nonuniform pattern, with the proportion of plus-end-distal MTs increasing progressively with distance from the cell body (see Fig. $2 e$ for data).

Because plus- and minus-end-distal MTs were distributed differentially, we hypothesized that MTs of different orientations may arise in the processes at different stages of their development, similar to dendrites. To investigate this possibility, we analyzed the MT arrays of five nascent processes, all of which were shorter than $10 \mu \mathrm{m}$. In all of these processes, the MTs were predominantly plus end distal (Fig. $3 a, b$; see legend for data), indicating that the nonuniform MT array of the mature process arises from an antecedent containing predominantly plus-end-distal MTs. As mentioned earlier, MT bundles are present within the cell bodies of many infected cells. They are particularly abundant in cells that had not yet extended processes and those with short nascent processes. Analyses of five of these were performed as well. Although there was no vantage point from which to judge the MTs of the bundles as plus or minus end distal, in all five cases, the MTs within the bundles were predominantly of the same orientation (Fig. 3a,c; see legend for data). This suggests that bundling of uniformly oriented MTs occurs independently of process formation.

\section{Process formation in the absence of net MT assembly}

The simplest interpretation of these data are that process formation involves the transport of MTs with specific orientations from the cell body into the developing processes. An alternate possibility, however, is that ectopic expression of the $\mathrm{N}$-terminal CHO1/MKLP1 fragment induces process formation by way of large-scale MT assembly and that this results in the nonuniform MT pattern within the processes. To investigate this possibility, we infected cells in the presence of $10 \mathrm{~nm}$ vinblastine to prohibit any significant MT assembly from occurring during process formation. Cells grown under these conditions elaborated thick tapering processes in a manner similar to that of undrugged cells. Figure $4 a-c$ shows immunofluorescence images of the MT polymer within an uninfected cell, an infected cell with no drug, and a cell infected in the presence of vinblastine. Quantitative fluorescence analyses were performed on 10 representative cells from of each of these culture conditions. The results of these analyses, group mean \pm SD in AFUs, were as follows: control cells, $1400 \pm 500$ AFUs; infected cells without vinblastine, $4100 \pm 1000$ AFUs; infected cells with vinblastine, $1100 \pm 350$ AFUs. There was a threefold increase in polymer levels within the undrugged infected 

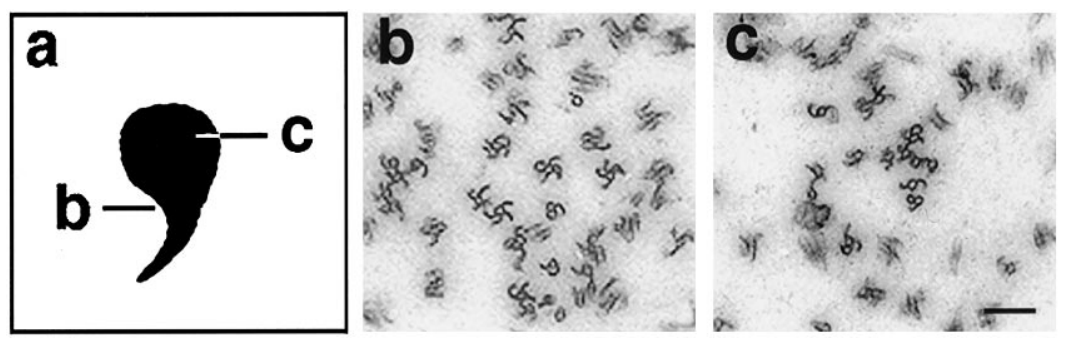

Figure 3. $a$, Tracing of a cell that extended a short nascent process after being induced to express the $\mathrm{N}$-terminal half of CHO1/MKLP1. Arrows indicate points at which MT polarity was assessed. $b$, Electron micrograph of the MT array at the point indicated in $a . c$, Electron micrograph of an MT bundle observed just under the plasma membrane in the cell body. As noted in the legend to Figure 2, clockwise hooks indicate the plus ends of MTs, whereas counterclockwise hooks indicate the minus ends of MTs. In $b$, observations were made from the vantage point of the distal end of the process, therefore clockwise hooks indicate MTs with their plus ends distal to the cell body, whereas counterclockwise indicate MTs with their minus ends distal to the cell body. In $c$, MT bundles were clustered just beneath the plasma membrane and, hence, there was no point of reference from which to judge MT polarity orientation. Therefore, hooks indicate the degree of uniformity of the polarity of bundled MTs. In total, five nascent processes between 3 and $10 \mu \mathrm{m}$ in length were analyzed at one point near the cell body, and the results were as follows: (1) 68 clockwise $/ 12$ counterclockwise, $85 \%$ plus end distal; (2) 61 clockwise/14 counterclockwise, $81 \%$ plus end distal; (3) 37 clockwise $/ 6$ counterclockwise, $86 \%$ plus end distal; (4) 42 clockwise $/ 10$ counterclockwise, $81 \%$ plus end distal; (5) 106 clockwise $/ 8$ counterclockwise, $93 \%$ plus end distal. Five MT bundles were analyzed from three different cell bodies: (1) 11 clockwise/ 0 counterclockwise, $100 \%$ uniformity; (2) 0 clockwise/14 counterclockwise, $100 \%$ uniformity; (3) 2 clockwise/17 counterclockwise, $89 \%$ uniformity; (4) 11 clockwise/1 counterclockwise, $92 \%$ uniformity; (5) 6 clockwise/ 1 counterclockwise, $86 \%$ uniformity. Scale bars: $a, 4 \mu \mathrm{m} ; b, c, 0.125 \mu \mathrm{m}$.

cells relative to the control cells, but no increase in the infected drug-treated cells. Nevertheless, similar to the undrugged infected cells, there was a diminution of polymer from the cell body and a concomitant accumulation of polymer in the developing processes. In MT polarity analyses on the midregions of five such processes, $54 \%, 45 \%, 47 \%, 44 \%$, and $37 \%$ of the hooks were counterclockwise (see Fig. 4, inset). Thus, MTs of both orientations arose within the processes in the absence of new MT assembly. These results demonstrate that the transport of preassembled MTs is sufficient for the establishment of the nonuniform MT pattern within the developing processes.

\section{DISCUSSION}

Several different cell types including neurons, glia, and Sertoli cells extend elongate cellular processes containing arrays of MTs that are highly organized but not attached to a discrete nucleating structure. Most efforts at identifying the molecules that organize these MTs have focused on fibrous MAPs that principally function to stimulate MT bundling and assembly. When expressed ectopically in Sf9 cells, these MAPs increase MT levels and induce the formation of processes, but the MTs within the processes have the same uniformly plus-end-distal polarity pattern regardless of whether tau, an axon-enriched MAP, or MAP2, a dendriteenriched MAP, is expressed (Baas et al., 1991; Chen et al., 1992; LeClerc et al., 1993). These observations suggest that stimulating MT assembly and bundling is not sufficient to establish the characteristic nonuniform polarity pattern of neuronal dendrites. The present study and its predecessor (Kuriyama et al., 1994) are the first to document that expression of a molecular motor also induces Sf9 cells to form MT-rich processes. Interestingly, the processes induced by expression of the $\mathrm{N}$-terminal half of $\mathrm{CHO} 1 /$ MKLP1 have a smooth tapering morphology that is far more reminiscent of a dendrite than the morphology of processes induced by MAP expression. Moreover, the MTs within the processes have a nonuniform pattern of polarity orientation remarkably similar to that observed in the dendrite. This nonuniform polarity pattern is established by the sequential addition of plusend-distal MTs followed by minus-end-distal MTs, the same sequence observed during dendritic development (Baas et al., 1989).
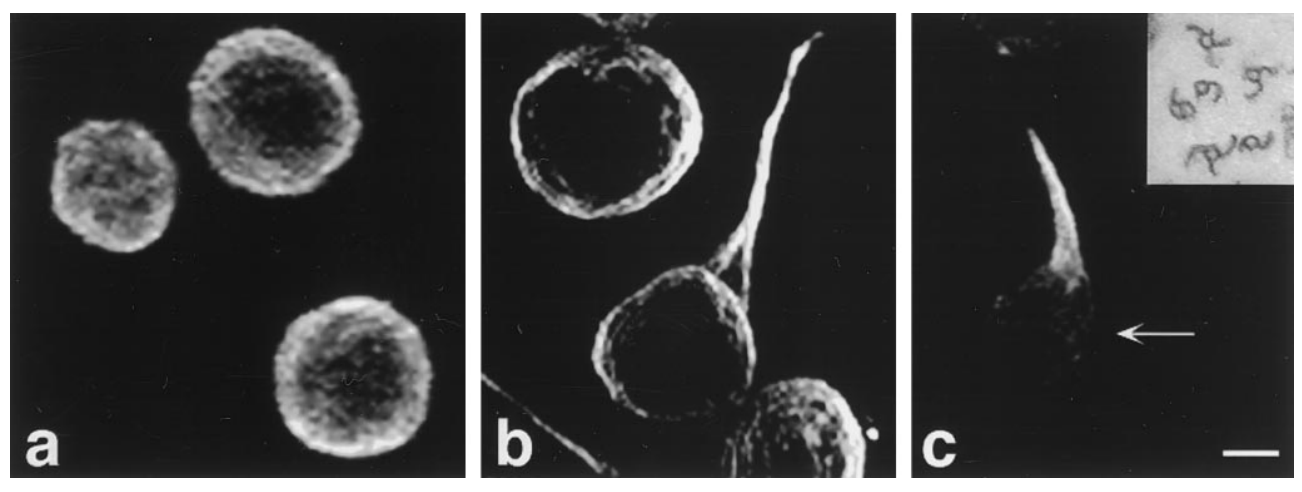

Figure 4. $a$, Uninfected Sf9 cells immunostained for MTs. $b$, Immunostain for MTs in Sf9 cells $3 \mathrm{~d}$ after infection with baculovirus construct encoding $\mathrm{N}$-terminal half of CHO1/MKLP1. $c$, Immunostain for MTs in an infected $\mathrm{Sf} 9$ cell that developed in the presence of $10 \mathrm{~nm}$ vinblastine. Arrow shows the cell body, which is nearly devoid of MTs. Inset, The MT array at the midpoint of a process that developed in the presence of vinblastine. Clockwise hooks indicate MTs with their plus ends distal to the cell body, whereas counterclockwise hooks indicate MTs of the opposite orientation. Approximately half of the MTs are of each orientation. Scale bar: $a-c, 9 \mu \mathrm{m}$; inset, $0.3 \mu \mathrm{m}$. 
These observations demonstrate that a molecular motor has the capacity to organize a distinctly dendritic pattern of MT organization.

\section{Mechanisms of motor-induced MT organization}

It may at first appear puzzling that a kinesin-related protein has the capacity to organize MTs, given that kinesins generally are thought to regulate organelle transport. Indeed, expression in Sf9 cells of a variety of other kinesin-related proteins has not resulted in the formation of processes or in any detectable change in MT organization (see Noda et al., 1995). However, the motor studied here, $\mathrm{CHO} 1 / \mathrm{MKLP} 1$, is different from these in that its primary function is to mediate interactions between MTs in the mitotic spindle. Experimental evidence suggests that CHO1/MKLP1 transports minus ends of MTs toward plus ends of other MTs in the midzone, resulting in spindle elongation during anaphase B. This transport uses ATP-sensitive MT-binding domains within the $\mathrm{N}$-terminal region of the molecule. Recent studies using a MT sedimentation assay indicate that the $\mathrm{N}$-terminal region also contains ATP-insensitive MT-binding domains that presumably are involved in MT bundling (Kuriyama et al., 1994). In support of this conclusion is the observation that expression in Sf9 cells of the $\mathrm{N}$-terminal half of the molecule results in the formation of MT bundles throughout the cell body. Our analyses indicate that the MTs within these bundles are uniformly oriented with regard to their polarity. It is unclear at this point whether this uniformity results directly from the bundling properties of the motor fragment, from the sliding apart of bundles of oppositely oriented MTs, or from endogenous factors within Sf9 cells. Some support for the first possibility derives from the localization of CHO1/ MKLP1 along uniformly oriented MTs emanating from the same centrosome early during mitotic progression.

Based on the properties of CHO1/MKLP1 to translocate MTs of opposite orientation relative to one another, it seems reasonable that the transport properties of the motor establish the nonuniform MT pattern within the processes. However, the finding that the MT polymer mass of infected cells is three times greater than that in controls indicates that infection and, possibly, high concentrations of the expressed CHO1/MKLP1 fragment somehow result in net MT assembly during process outgrowth. This finding led to some uncertainty as to whether MT transport in fact had generated nonuniform MT polarity orientation or whether the increase in MT assembly was the critical factor. To address this issue, we infected Sf9 cells in the presence of the anti-MT drug vinblastine at a concentration that suppresses net MT assembly. As expected, the processes formed under these conditions contained fewer MTs than those formed without vinblastine. Nevertheless, the processes contained essentially the same proportions of MTs of each orientation, indicating that net MT assembly is not required for the generation of nonuniform MT polarity orientation in these processes. Furthermore, process formation always was accompanied by a decrease of MT polymer in the cell body and a concomitant increase in the developing process, whether or not the infection was carried out in the presence of the drug. These findings suggest strongly that the generation of the nonuniform MT polarity pattern within the processes is established via the transport of specifically oriented MTs from the cell body.

We propose that the MT array of the motor-induced processes is established as follows. First, the CHO1/MKLP1 fragment bundles MTs of the same orientation within the cell body. In those cases in which the plus ends of the MTs are oriented toward the periphery, the MT bundles may participate in the formation of nascent pro-

\section{Mitotic Spindle}

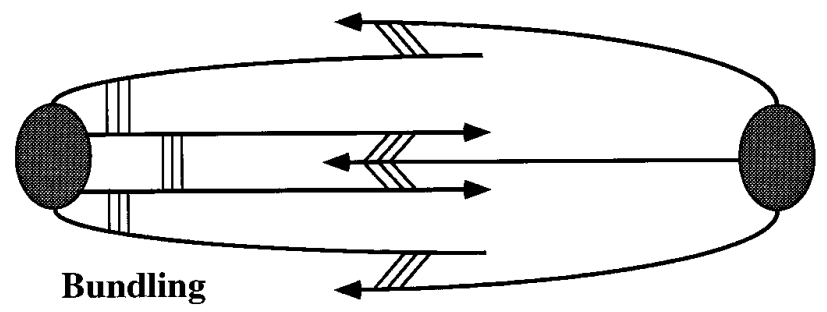

Transport

\section{Process Formation}

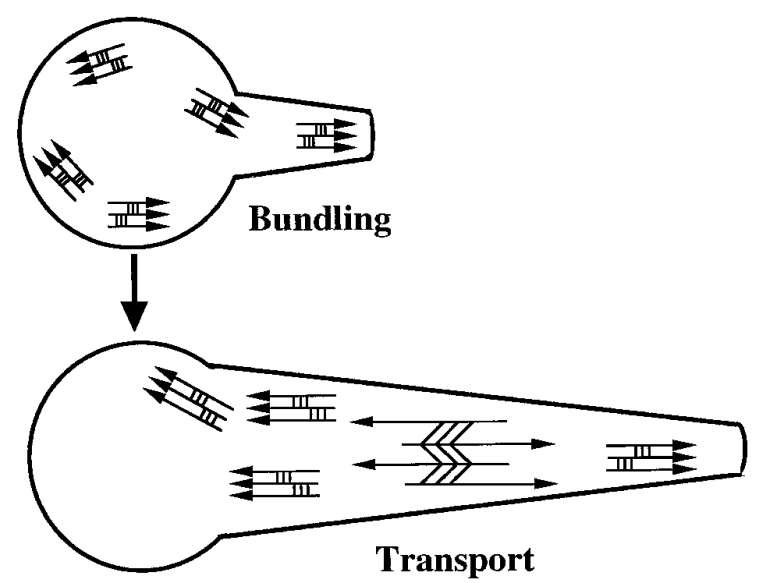

Figure 5. Schematic showing our model for the effects of CHO1/MKLP1 on MT bundling and transport in the mitotic spindle and of the N-terminal fragment during Sf9 process formation. Line segments with arrowheads depict MTs. Arrowheads indicate plus ends of MTs. Thinner lines connecting MTs represent CHO1/MKLP1 in the mitotic spindle and its N-teminal fragment in Sf9 cells. Three thin lines perpendicular to the MTs represent the proposed property of the motor to bundle parallel MTs. Three thin lines diagonal to the MTs represent the documented property of $\mathrm{CHO}$ / MKLP1 to transport antiparallel MTs against one another. CHO1/ MKLP1 localizes early during mitosis to the regions of each half-spindle where it bundles parallel MTs and then, subsequently, to the midregion where it transports antiparallel MTs against one another. During Sf9 process formation, the N-terminal fragment induces the formation of short bundles of uniformly oriented MTs. Initially, these bundles form throughout the cell body but with time cluster near the cell periphery. Then the MTs, if oriented with their plus ends facing the periphery, are able to participate in process initiation. These plus-end-distal MTs act as a substrate for the CHO1/MKLP1 fragment to transport MTs into elongating processes with their minus ends leading.

cesses. Second, the CHO1/MKLP1 fragment transports MTs with their minus ends leading from the cell body into these nascent processes. During this phase of process development, the motor uses the plus-end-distal MTs within the nascent processes as a substrate along which to transport the minus-end-distal MTs (see Fig. 5). The idea that both MT bundling and MT transport are served by the $\mathrm{N}$-terminal half of the molecule is consistent with the fact that this portion of the molecule contains both classes of MT binding sites present on the full-length molecule.

As the process continues to develop, it is necessary for the plus-end-distal MTs to translocate down the processes to pro- 
vide a "moving substrate" along which the motor can continue to transport the minus-end-distal MTs. This is apparent from our finding that plus-end-distal MTs are enriched distally in the process, whereas minus-end-distal MTs are enriched proximally. The latter probably become stranded once their substrate, the plus-end-distal MTs, have cleared the proximal region. Interestingly, a similar enrichment of minus-end-distal MTs has been observed in the proximal regions of dendrites grown under conditions that inhibit the replenishment of plusend-distal MTs (Sharp et al., 1995). It is unlikely that the CHO1/MKLP1 fragment is directly responsible for the transport of plus-end-distal MTs down the process. We suspect that the transport of MT bundles with their plus ends leading toward cell periphery and into nascent processes involves machinery endogenous to Sf9 cells. Indeed, previous studies have shown that Sf9 cells are able to orient MTs with plus ends distal to the cell body in the absence of any ectopic motor expression. Expression in these cells of fibrous MAPs such as tau, which stimulate the formation of bundles of randomly oriented MTs in vitro (Brandt and Lee, 1994), results in the formation of processes containing bundles of uniformly plus-end-distal MTs.

\section{CONCLUSION}

The present study documents that a portion of the CHO1/MKLP1 molecule is able to organize MTs into arrays with a distinctly dendritic pattern of organization. These findings are consistent with previous data suggesting that MT transport establishes the nonuniform polarity pattern of dendritic MTs (Sharp et al., 1995). CHO1/MKLP1 appears to be ubiquitous within vertebrate cells undergoing mitosis, but it is notable that these cells do not use CHO1/MKLP1 for the generation of cellular processes. The same generally is true for $\mathrm{Sf} 9$ cells induced to express full-length $\mathrm{CHO} 1 /$ MKLP1, raising the possibility that the C-terminal half of the molecule serves a regulatory function. In particular, modifications of the C-terminal domain may alter the capacity of the motor to participate in process formation. Thus, it is conceivable that the relevant motor in dendrites is a modified variant of $\mathrm{CHO} 1 /$ MKLP1, an alternatively spliced product of the same gene, or a different motor with properties similar to the $\mathrm{N}$-terminal half of CHO1/MKLP1. Current efforts are directed at identifying novel MT motors that are expressed during dendritic development and testing their potential roles in the transport and organization of dendritic MTs.

A broader point to be made is that motor-driven MT transport may be an important means by which many cell types organize MTs into sophisticated patterns of polarity orientation. If this is correct, then the expression of specific molecular motors may be critical for the establishment of such arrays and, in turn, for initiating developmental milestones such as the differentiation of axons and dendrites. Our studies suggest that some of these motors may be similar to those that regulate MT interactions during mitosis. We find provocative the idea that MT behaviors during mitosis and process formation may be variations on the same theme.

\section{REFERENCES}

Baas PW, Ahmad FJ (1993) The transport properties of axonal microtubules establish their polarity orientation. J Cell Biol 120:1427-1437.

Baas PW, White LA, Heidemann SR (1987) Microtubule polarity reversal accompanies regrowth of amputated neurites. Proc Natl Acad Sci USA 84:5272-5276.

Baas PW, Deitch JS, Black MM, Banker GA (1988) Polarity orientation of microtubules in hippocampal neurons: uniformity in the axon and nonuniformity in the dendrite. Proc Natl Acad Sci USA 85:8335-8339.

Baas PW, Black MM, Banker GA (1989) Changes in microtubule polarity orientation during the development of hippocampal neurons in culture. J Cell Biol 109:3085-3094.

Baas PW, Pienkowski TP, Kosik KS (1991) Processes induced by tau expression in Sf9 cells have an axon-like microtubule organization. J Cell Biol 115:1333-1344.

Brandt R, Lee G (1994) Orientation, assembly and stability of microtubule bundles induced by a fragment of tau protein. Cell Motil Cytoskeleton 28:143-154.

Burton PR, Paige JL (1981) Polarity of axoplasmic microtubules in the olfactory nerve of the frog. Proc Natl Acad Sci USA 78:3269-3273.

Chen J, Kanai Y, Cowan NJ, Hirokawa N (1992) Projection domains of MAP-2 and tau determine spacings between microtubules in dendrites and axons. Nature 360:674-677.

Euteneuer U, McIntosh JR (1981) Structural polarity of kinetochore microtubules in PTK2 cells. J Cell Biol 98:338-345.

Heidemann SR, McIntosh JR (1980) Visualization of the structural polarity of microtubules. Nature 286:517-519.

Heidemann SR, Landers JM, Hamborg MA (1981) Polarity orientation of axonal microtubules. J Cell Biol 91:661-665.

Hoyt MA (1994) Cellular roles of kinesin and related proteins. Curr Opin Cell Biol 6:63-68.

Knops J, Kosik KS, Lee G, Pardee JD, Cohen-Gould L, McConlogue L (1991) Overexpression of tau in a nonneuronal cell induces long cellular processes. J Cell Biol 114:725-733.

Kuriyama R, Dragas-Granoic S, Maekawa T, Vassilev A, Khodjakov A, Kobayashi H (1994) Heterogeneity and microtubule interaction of the $\mathrm{CHO} 1$ antigen, a mitosis-specific kinesin-like protein. Analysis of subdomains expressed in insect Sf9 cells. J Cell Sci 107:3485-3499.

LeClerc N, Kosik KS, Cowan N, Pienkowski TP, Baas PW (1993) Process formation in Sf9 cells induced by the expression of a microtubuleassociated protein 2c-like construct. Proc Natl Acad Sci USA 90:6233-6227.

Maekawa T, Leslie R, Kuriyama R (1991) Identification of a minus end-specific microtubule-associated protein located at the mitotic poles in cultured mammalian cells. Eur J Cell Biol 54:255-267.

McIntosh JR (1994) The roles of microtubules in chromosome movement. In Microtubules (JS Hyams and CW Lloyd, eds), pp 413-434. New York: Wiley-Liss.

Nislow C, Sellitto C, Kuriyama R, McIntosh JR (1990) A monoclonal antibody to a mitotic microtubule-associated protein blocks mitotic progression. J Cell Biol 111:511-522.

Nislow C, Lombillo VA, Kuriyama R, McIntosh JR (1992) A plus-enddirected motor that moves anti-parallel microtubules in vitro localizes in the interzone of mitotic spindles. Nature 359:543-547.

Noda Y, Sato-Yoshitake R, Kondo S, Nangaku M, Hirokawa N (1995) KIF2 is a new microtubule-based anterograde motor that transports membranous organelles distinct from those carried by kinesin heavy chain or KIF3A/B. J Cell Biol 129:157-167.

Sellito C, Kuriyama R (1988) Distribution of a matrix component of the midbody during the cell cycle in Chinese hamster ovary cells. J Cell Biol 106:431-439.

Sharp DJ, Yu W, Baas PW (1995) Transport of dendritic microtubules establishes their nonuniform polarity orientation. J Cell Biol 130: 93-104. 